Review

\title{
Immunotherapy in colorectal cancer: current achievements and future perspective
}

\author{
Ahui Fan*, Boda Wang*, Xin Wang, Yongzhan Nie, Daiming Fan, Xiaodi Zhao ${ }^{\bowtie}$ and Yuanyuan Lu ${ }^{\varpi}$ \\ State Key Laboratory of Cancer Biology and National Clinical Research Center for Digestive Diseases, Xijing Hospital of Digestive Diseases, Fourth Military \\ Medical University, Xi'an, Shaanxi 710032, China. \\ *These authors contributed equally to this work. \\ $\triangle$ Corresponding authors: luyuandreamer@aliyun.com; leedyzhao@fmmu.edu.cn. \\ (c) The author(s). This is an open access article distributed under the terms of the Creative Commons Attribution License (https://creativecommons.org/licenses/by/4.0/). \\ See http://ivyspring.com/terms for full terms and conditions.
}

Received: 2021.06.22; Accepted: 2021.08.06; Published: 2021.09.03

\begin{abstract}
Following dramatic success in many types of advanced solid tumors, interest in immunotherapy for the treatment of colorectal cancer (CRC) is increasingly growing. Given the compelling long-term durable remission, two programmed cell death 1 (PD-1)-blocking antibodies, pembrolizumab and nivolumab (with or without Ipilimumab), have been approved for the treatment of patients with metastatic colorectal cancer (mCRC) that is mismatch-repair-deficient and microsatellite instability-high (dMMR$\mathrm{MSI}-\mathrm{H}$ ). Practice-changing results of several randomized controlled trials to move immunotherapy into the first-line treatment for MSI-H metastasis cancer and earlier stage were reported successively in the past 2 years. Besides, new intriguing advances to expand the efficacy of immunotherapy to $\mathrm{mCRC}$ that is mismatch-repair-proficient and low microsatellite instability (PMMR-MSI-L) demonstrated the potential benefits for the vast majority of $\mathrm{mCRC}$ cases. Great attention is also paid to the advances in cancer vaccines and adoptive cell therapy (ACT). In this review, we summarize the above progresses, and also highlight the current predictive biomarkers of responsiveness in immunotherapy with broad clinical utility.
\end{abstract}

Key words: colorectal cancer; immunotherapy; immune checkpoint inhibitors; adoptive cell therapy; cancer vaccines; biomarkers

\section{Introduction}

Colorectal cancer (CRC) is the third most common cancer and the second cause of cancer-related death worldwide. It is considered as a global health issue with an urgent unmet need of new therapeutic strategies [1]. Although screening has reduced the incidence and mortality, approximately $25 \%$ CRC patients present with advanced stage disease at the time of diagnosis, and in patients with early-stage disease nearly $25 \%-50 \%$ will develop metastasis [2-4]. The patients with oligometastatic disease after tumor resection and systematic therapy have 5-year survival rates of $40 \%$, whereas the patients with metastatic colorectal cancer (mCRC) are only about 20\% [5-8]. While the benefits of chemotherapy and targeted therapy have reached a plateau, it is urgent to develop new effective treatment strategy to improve survival outcome.
Immunotherapy is aiming at harnessing the immune system to battle cancer. Immune checkpoint inhibitors (ICIs), modulating the interaction of T cells, antigens-presenting cells (APCs) and tumor cells to help unleash suppressed immune responses, emerged as a very effective therapy for patients with mCRC that is mismatch-repair-deficient (dMMR) or microsatellite instability-high (MSI-H) (termed dMMR/MSI-H mCRC). Owing to the efficacious, stable and durable responses, pembrolizumab and nivolumab (with or without Ipilimumab) were approved by US Food and Drug Administration (FDA) for the treatment of these patients. However, mCRC is characterized by insufficient mutated tumor antigens [9], thus the main challenge is to provide the benefit of immunotherapy for the vast majority of mCRC patients that are mismatch-repair-proficient 
(pMMR) or microsatellite-stable (MSS) or low microsatellite instability (MSI-L) (termed pMMR/ MSS/MSI-L mCRC). In this review, we summarize the present evidences supporting the use of ICIs in $\mathrm{CRC}$, focus on the recent advances in expanding the scope of ICIs in pMMR/MSS/MSI-L CRC, sum up the research progress of other anticipated immunoapproaches and highlight the emerging biomarkers for prediction of responsiveness to immunotherapy.

\section{ICIs-based immunotherapy}

\section{Clinical Application of ICls}

\section{Biomarkers: MMR and MSI}

Mismatch repair (MMR) plays a critical role in maintaining DNA fidelity [10]. By quantification of the MMR proteins MLH1, MSH2, MSH6 or PMS2 using immunohistochmical staining, CRC can be divided into dMMR or pMMR CRC [11]. Notably, the change of MMR status can contribute to the change of microsatellite length named microsatellite instability (MSI) due to insertions and deletions, which can be detected accurately by PCR or next-generation sequencing [11]. In dMMR-MSI-H tumors, major histocompatibility complex (MHC) class I-peptide complexes present on the surface of tumor cells, including mutated peptides which could be recognized as neoantigens and subsequently promote immune cells priming and infiltration. In particular, CD8+ tumor-infiltrating lymphocytes (TILs), T helper 1 (TH1) CD4+T cells and macrophages migrate into the tumor microenvironment, and elicit IFNY secretion and anti-tumor effect. Meanwhile, dMMRMSI-H tumor cells continuously upregulate $\mathrm{T}$ cell inhibitory ligands to promote immune escape, such as PD-L1, CD80 and CD86 of the B7 family [12-16].

Nearly $15 \%$ of all CRCs are dMMR-MSI-H, and the percentage is associated with tumor stage [17]. Approximately $5 \% \sim 20 \%$ of stage 2 and $11 \%$ of stage 3 tumors are dMMR-MSI-H, whereas, the percentage is only $5 \%$ in stage 4 [18]. Moreover, dMMR-MSI-H is a prognostic biomarker for patients of different stages [18-21]. In stage 2 and stage 3, patients with dMMRMSI-H tumors have much better prognosis than those with pMMR-MSI-L tumors. Remarkably, stage 4 patients with dMMR-MSI-H show dismal prognosis, but have good response to immune checkpoint blockade [22].

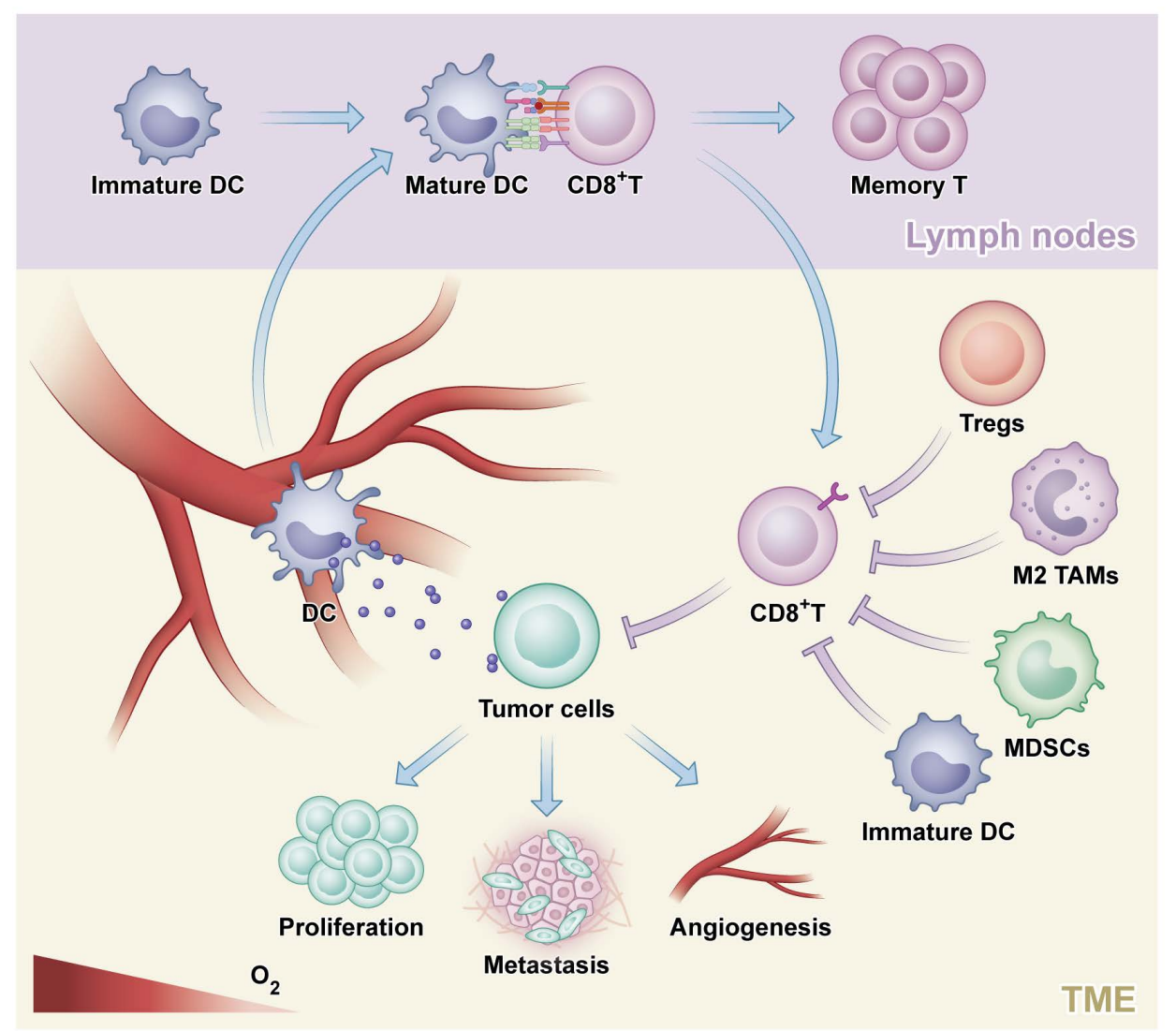

Figure 1. The process of an antitumor response. Tumor cells generate and release neoantigens which could be phagocytosed by dendritic cells (DC). Next, DC mediated presentation of tumor-specific antigens to CD8+ T cells and CD4+ helper T typel cells which could enhance the effects of CD8+ $T$ cells on killing tumor and promote the generation of tumor-specific activated $T$ cells and memory $T$ cells. Finally, tumor cells are destructed by effector $T$ cells, which could be inhibited by regulatory $T$ cells (Tregs), M2-polarized tumor-associated macrophage (M2 TAMs), myeloid-derived suppressor cells (MDSCs) and immature DC. 


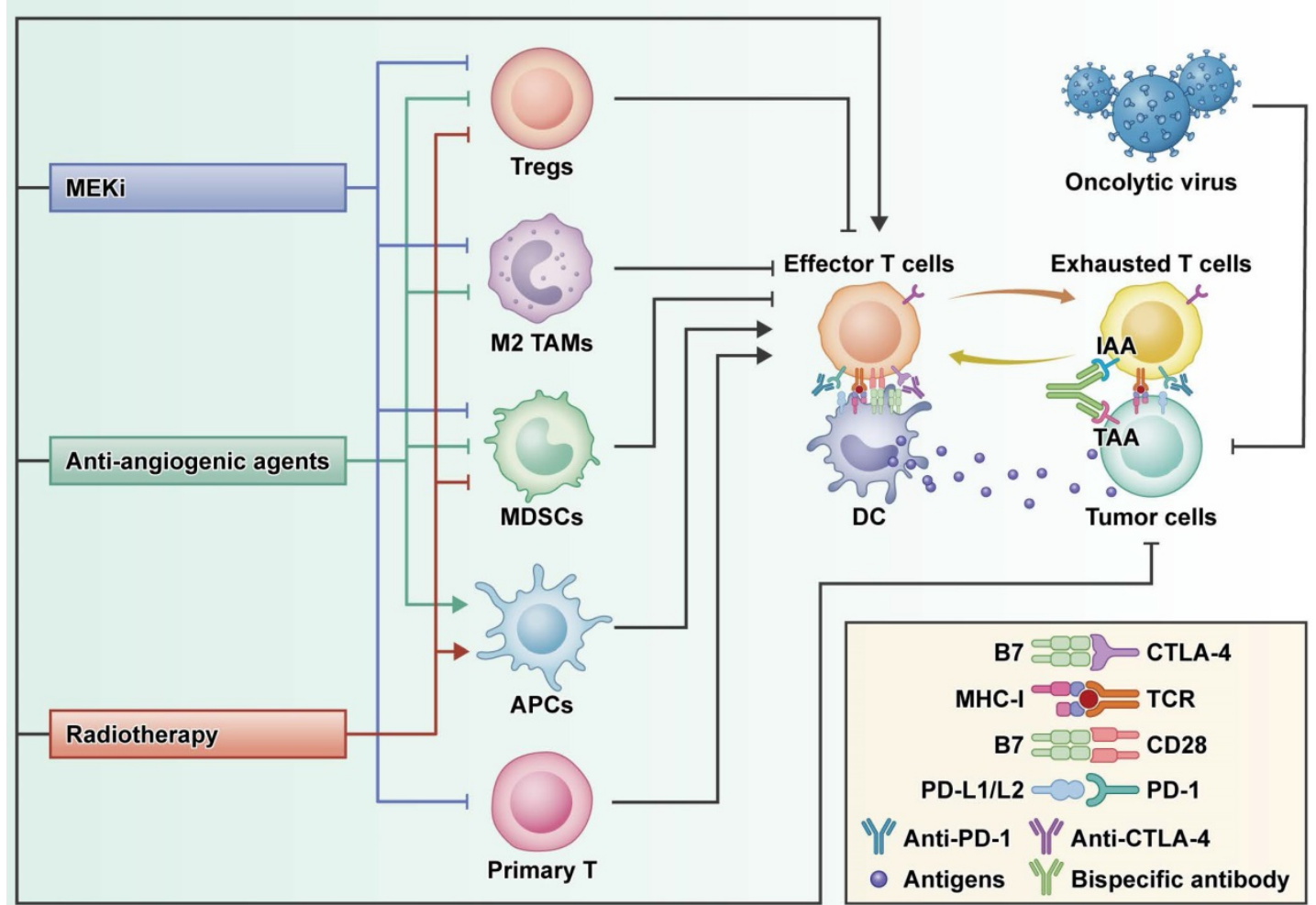

Figure 2. The rationale of combination strategies to overcome primary ICI resistance in CRC. MEKi: MEK inhibitor; Tregs: regulatory T cells; TAMs: tumorassociated macrophage; MDSCs: myeloid- derived suppressor cells; APCs: antigen presenting cells; DC: dendritic cells; IAA: immune-associated antigen; TAA: tumor-associated antigen.

Table 1. Ongoing trials in AMMR-MSI-H CRC

\begin{tabular}{|c|c|c|c|c|c|}
\hline Treatment & $\begin{array}{l}\text { Clinicaltrials.gov } \\
\text { Identifier }\end{array}$ & Phase & $\begin{array}{l}\text { Study treatment } \\
\text { groups }\end{array}$ & $\begin{array}{l}\text { Primary } \\
\text { endpoint }\end{array}$ & $\begin{array}{l}\text { Recruitment } \\
\text { status }\end{array}$ \\
\hline \multirow[t]{2}{*}{ First-line } & NCT02563002 & III & $\begin{array}{l}\text { Pembrolizumab } \\
\text { versus } \\
\text { standard-of-care } \\
\text { chemotherapy }\end{array}$ & $\begin{array}{l}\text { PFS, OS, } \\
\text { ORR }\end{array}$ & $\begin{array}{l}\text { Active, not } \\
\text { recruiting }\end{array}$ \\
\hline & NCT02060188 & II & $\begin{array}{l}\text { Nivolumab } \pm \\
\text { ipilimumab or } \\
\text { daratumumab or } \\
\text { anti-LAG3 } \\
\text { antibody }\end{array}$ & ORR & $\begin{array}{l}\text { Active, not } \\
\text { recruiting }\end{array}$ \\
\hline Adjuvant & NCT02912559 & III & $\begin{array}{l}\text { Adjuvant } \\
\text { atezolizumab + } \\
\text { FOLFOX versus } \\
\text { FOLFOX alone }\end{array}$ & DFS & Recruiting \\
\hline \multirow[t]{3}{*}{ Neoadjuvant } & NCT03026140 & II & $\begin{array}{l}\text { Nivolumab }+ \\
\text { ipilimumab } \pm \\
\text { celecoxib }\end{array}$ & Safety & Recruiting \\
\hline & NCT02948348 & $\mathrm{Ib} / \mathrm{II}$ & $\begin{array}{l}\text { Capecitabine }+ \\
\text { radiation }+ \\
\text { nivolumab }+ \\
\text { surgical therapy }\end{array}$ & pCR & Unknown \\
\hline & NCT02921256 & II & $\begin{array}{l}\text { Pembrolizumab/ } \\
\text { veliparib + } \\
\text { chemotherapy + } \\
\text { radiotherapy }\end{array}$ & $\begin{array}{l}\text { NAR } \\
\text { score }\end{array}$ & $\begin{array}{l}\text { Active, not } \\
\text { recruiting }\end{array}$ \\
\hline
\end{tabular}

PFS, progression-free survival; OS, overall survival; ORR, overall response rate; DFS, disease-free survival; $p C R$, pathological complete response; NAR, Change in neoadjuvant rectal cancer (NAR) score.

\section{Approval for the second-line treatment in dMMR-MSI-H mCRC}

Pembrolizumab and nivolumab (with or without Ipilimumab) are clinically approved in 2017 as the second-line treatment for patients with dMMR-MSI-H
mCRC. KEYNOTE016 (NCT01876511) is a phase II trial using pembrolizumab to treat patients with refractory mCRC [23]. The overall response rate (ORR) and disease control rate (DCR) were $50 \%$ and $89 \%$ for dMMR-MSI-H mCRC compared with $0 \%$ and $16 \%$ for pMMR-MSI-L, respectively. CheckMate142 (NCT02060188) is a phase II trial using nivolumab, with or without ipilimumab in patients with dMMRMSI-H mCRC [24]. In a median follow-up duration of 13.4 months, $55 \%$ and $80 \%$ of 119 patients in the nivolumab monotherapy arm showed ORR and DCR of 12 or more weeks, respectively. The 12-month progression free survival (PFS) and overall survival (OS) were $71 \%$ and $85 \%$, respectively $[25,26]$.

\section{Exploration of ICls in dMMR-MSI-H CRC}

Given the cogent efficacy of immunotherapy in dMMR-MSI-H mCRC, the interest in exploring the potential of immunotherapy for the first line treatment of CRC patients, even in earlier-stage, is increasingly growing. The promising data of several randomized clinical trials has elicited tremendous excitement [27-29].

\section{First-line treatment}

KEYNOTE177 trial has attracted great attention, as an international randomized phase III study to assess pembrolizumab monotherapy verse standard 
of care in previously untreated stage 4 dMMR-MSI-H CRC patients [30]. The primary end points are PFS and OS, and the secondary end point is ORR. Practicechanging results supported first-line pembrolizumab monotherapy for dMMR-MSI-H mCRC [31]. The PFS and ORR were 16.5 months and $43.8 \%$, respectively, in the pembrolizumab group as compared with 8.2 months (hazard ratio 0.60 ) and $33.1 \%$, respectively, in the combined chemotherapy and bevacizumab/ cetuximab group. The median DOR in the control group is 10.6 months while it had not been reached in the pembrolizumab group. With respect to safety, the pembrolizumab group showed less treatment-related adverse events in patients with grade 3 or higher. Based on the compelling data, FDA approved pembrolizumab for first-line treatment of dMMR/ MSI-H mCRC in Jun.2020. Additionally, after a median follow-up duration of 44.5 months (36.0-60.3) with pembrolizumab vs 44.4 months (36.2-58.6) with chemotherapy, final analysis demonstrated that the ORR of the pembrolizuman increased from $43.8 \%$ to $45 \%$.

The efficacy and safety of first-line treatment with combined nivolumab and low-dose ipilimumab in patients of dMMR-MSI-H tumors were evaluated in CheckMate142 [32]. After a median follow-up period of 13.8 months, the ORR and DCR were $60 \%$ and $84 \%$, respectively, and the rate of complete response (CR) was 7\%. An updated analysis of patients followed-up for a median of 29.0 months presented in the 2020 demonstrated a similar DCR $84 \%$. Notably, the rate of CR increased from $7 \%$ at 13.8 months to $13 \%$ at 29.0 months, and the ORR increased from $60 \%$ to $69 \%$ [32]. Compared with pembrolizuman monotherapy in KEYNOTE177, the combination of nivolumab and ipilimumab showed better efficacy and safety.

Table 2. Combination trials in pMMR-MSI-L CRC

\begin{tabular}{|c|c|c|c|c|c|c|}
\hline Combination & $\begin{array}{l}\text { Clinicaltrials.gov } \\
\text { Identifier }\end{array}$ & Phase & Checkpoint inhibitors & $\begin{array}{l}\text { Other intervention/ } \\
\text { treatment (target) }\end{array}$ & $\begin{array}{l}\text { Primary } \\
\text { endpoint }\end{array}$ & $\begin{array}{l}\text { Recruitment } \\
\text { status }\end{array}$ \\
\hline \multirow[t]{6}{*}{ Radiation } & NCT02888743 & II & Duvalumab (PD-1) + Tremelimumab (CTLA-4) & - & ORR & $\begin{array}{l}\text { Active, not } \\
\text { recruiting }\end{array}$ \\
\hline & NCT03007407 & II & Duvalumab (PD-1) + Tremelimumab (CTLA-4) & - & ORR & Completed \\
\hline & NCT03104439 & II & Nivolumab (PD-1) + Ipilimumab (CTLA-4) & - & DCR & Recruiting \\
\hline & NCT04575922 & II & Nivolumab (PD-1) + Ipilimumab (CTLA-4) & - & DCR & $\begin{array}{l}\text { Not yet } \\
\text { recruiting }\end{array}$ \\
\hline & NCT04030260 & II & Nivolumab (PD-1) & Regorafenib (Multikinase) & PFS & Recruiting \\
\hline & NCT04535024 & II & Sintilimab (PD-1) & $\begin{array}{l}\text { Stereotactic Ablative } \\
\text { Radiotherapy }\end{array}$ & ORR & Recruiting \\
\hline \multirow[t]{5}{*}{$\begin{array}{l}\text { MEK } \\
\text { inhibitor }\end{array}$} & NCT02060188 & II & Nivolumab (PD-1) \pm Ipilimumab (CTLA-4) & Cobimetinib (MEK) & ORR & $\begin{array}{l}\text { Active, not } \\
\text { recruiting }\end{array}$ \\
\hline & NCT03271047 & I/II & Nivolumab (PD-1) \pm Ipilimumab (CTLA-4) & Binimetinib (MEK) & DLTs/ORR & $\begin{array}{l}\text { Active, not } \\
\text { recruiting }\end{array}$ \\
\hline & NCT03377361 & I/II & Nivolumab (PD-1) \pm Ipilimumab (CTLA-4) & Tramatinib (MEK) & AEs, SAEs, ORR & $\begin{array}{l}\text { Active, not } \\
\text { recruiting }\end{array}$ \\
\hline & NCT02788279 & III & Atezolizumab (PD-L1) & $\begin{array}{l}\text { Cobimetinib (MEK) + } \\
\text { Regorafenib (multikinase) }\end{array}$ & OS, PFS, OR & Completed \\
\hline & NCT03428126 & II & Duvalumab (PD-1) & Tramatinib (MEK) & MTD, OR & $\begin{array}{l}\text { Active, not } \\
\text { recruiting }\end{array}$ \\
\hline Anti-angiogenic & NCT01633970 & I & Atezolizumab (PD-L1) & Bevacizumab (VEGF) & AEs, DLTs, MTD & Completed \\
\hline \multirow[t]{2}{*}{ Agents } & NCT03406871 & $\mathrm{I} / \mathrm{II}$ & Nivolumab (PD-1) & Regorafenib (multikinase) & RD, MTD & $\begin{array}{l}\text { Active, not } \\
\text { recruiting }\end{array}$ \\
\hline & NCT04446091 & II & Carilizumab (PD-1) & $\begin{array}{l}\text { anti-angiogenic TKIs } \pm \\
\text { Irinotecan }\end{array}$ & ORR & Recruiting \\
\hline \multirow{3}{*}{$\begin{array}{l}\text { Bispecific } \\
\text { antibody }\end{array}$} & NCT02650713 & I & Atezolizumab (PD-L1) & RO6958688 (CEA - CD3) & AEs, DLTs, MTD & Completed \\
\hline & NCT03752398 & I & Ipilimumab (CTLA-4) & XmAb23104 (ICOS - PD-1) & AEs & Recruiting \\
\hline & NCT04429542 & I & Pembrolizumab (PD-1) & BCA101 (EGFR -TGF $\beta$ ) & AEs, SAEs, DLTs & Recruiting \\
\hline \multirow[t]{3}{*}{$\begin{array}{l}\text { Oncolytic } \\
\text { virus }\end{array}$} & NCT02636036 & I & Nivolumab (PD-1) & Enadenotucirev & $\begin{array}{l}\text { SAEs, DLTs, } \\
\text { MTD }\end{array}$ & Recruiting \\
\hline & NCT03206073 & I/II & Duvalumab (PD-1) + Tremelimumab (CTLA-4) & Pexa-Vec & AEs & $\begin{array}{l}\text { Active, not } \\
\text { recruiting }\end{array}$ \\
\hline & NCT04301011 & I/II & Pembrolizumab (PD-1) & TBio-6517 & AEs, MTD, MFD & Recruiting \\
\hline \multirow[t]{3}{*}{$\begin{array}{l}\text { Cancer } \\
\text { vaccine }\end{array}$} & NCT02981524 & II & Pembrolizumab (PD-1) & $\begin{array}{l}\text { GVAX + } \\
\text { Cyclophosphamide }\end{array}$ & ORR & Completed \\
\hline & NCT03050814 & II & Avelumab (PD-L1) & $\begin{array}{l}\text { Standard of care } \pm \\
\text { Ad-CEA }\end{array}$ & PFS & $\begin{array}{l}\text { Active, not } \\
\text { recruiting }\end{array}$ \\
\hline & NCT03639714 & I/II & Nivolumab (PD-1) + Ipilimumab (CTLA-4) & GRT-C901 + GRT-R902 & AEs, SAEs, DLTs & Recruiting \\
\hline Anti-EGFR & NCT03442569 & II & Nivolumab (PD-1) + Ipilimumab (CTLA-4) & Panitumumab (EGFR) & ORR & $\begin{array}{l}\text { Active, not } \\
\text { recruiting }\end{array}$ \\
\hline
\end{tabular}

ICIs, immunotherapy checkpoint inhibitors; PD-1, programmed cell death 1; CTLA-4, cytotoxic T-lymphocyte-associated protein 4; ORR, overall response rate; DCR, disease control rate; PFS, progression-free survival; DLTs, dose-limiting toxicities; AEs, number of adverse events; SAEs, number of serious adverse events; PD-L1, programmed cell death-Ligand1; OS, overall survival; MTD, maximum tolerated dose; MFD, maximum feasible dose; RD, recommended dose. 
Additionally, COMMIT (NCT02997228) is an undergoing randomized phase III trial of mFOLFOX6/bevacizumab combination with or without atezolizumab in 347 previously untreated patients with dMMR-MSI-H mCRC. The primary end point is PFS. The secondary end points are OS, ORR, DCR and incidence of adverse events [33]. The results are highly anticipated.

\section{Adjuvant and Neoadjuvant Therapy}

Adjuvant chemotherapy is indispensable for stage 3 CRC. Aiming to evaluate the potential efficacy of immunotherapy in adjuvant treatment, ATOMIC (NCT02912559), an ongoing phase III randomized controlled trial, enrolled 700 patients with stage 3 dMMR-MSI-H colon cancer. The patients were divided into 2 groups, which received 6 months of FOLFOX or FOLFOX plus atezolizumab for 6 months followed by atezolizumab alone for 6 months, respectively [34, 35]. The primary trial end point is disease-free survival (DFS), and the secondary end points include OS and incidence of adverse events. The results are highly anticipated.

High efficacy of neoadjuvant immunotherapy in early-stage CRC has been proved. NICHE (NCT03026140), a phase II exploratory study, enrolled 40 patients with stage 1-3 colon cancer, including 21 patients with dMMR tumors and 20 with pMMR tumors $[36,37]$. The primary endpoint is safety and feasibility. All patients with dMMR tumors were treated with a single dose of ipilimumab and two doses of nivolumab, successfully received surgery on schedule and met the primary endpoint. The exciting results showed that all of the 20 patients with dMMR tumors achieved pathological responses, with 19 major pathological responses (MPR) and 12 pathological complete responses ( $\mathrm{pCR}$ ).

NRG-GI002 (NCT02921256) is a randomized phase II trial to study the efficacy of veliparib or pembrolizumab when combined with chemotherapy and radiation therapy in patients with locally advanced rectal cancer (LARC) [38]. The primary endpoint is change in neoadjuvant rectal cancer (NAR) score, and the key secondary endpoints include OS, DFS, toxicity, pCR, clinical complete response (cCR), and sphincter sparing surgery (SSS). 185 patients were randomized to pembrolizumab $(n=90)$ or control $(n=95)$ group [39]. No improvement in NAR score was observed. The pCR was $29.4 \%$ in the pembrolizumab group compared with $31.9 \%$ in the control group, with the cCR $13.6 \%$ vs $13.9 \%$. The PFS and OS have not been reached.

VOLTAGE (NCT02948348) is a phase I b/II open-label single-arm study to investigate the safety and efficacy of sequential use of neoadjuvant immunotherapy after chemoradiotherapy with capecitabine and subsequent surgery in patients with locally advanced resectable rectal cancer [40]. 3 of 5 patients with dMMR-MSI-H tumors achieved pathological complete response and major pathological response [41].

Hopefully, neoadjuvant immunotherapy has the potential to become standard therapy of dMMRMSI-H CRC in the near future.

\section{Exploration of Immunotherapy in PMMR-MSI-L CRC}

Unlike dMMR-MSI-H CRC, pMMR-MSI-L tumors, which contribute to $95 \%$ of all mCRC cases, harbor a much lower mutation burden and poor recruitment of immune cells, leading to an unsatisfactory response to ICIs. With the deepening understanding of tumor mircoenvirnment of CRC, new discoveries and strategies of immune modulation have been explored and tested in pMMR-MSI-L $\mathrm{mCRC}$ patients to overcome primary ICI resistance.

\section{Combination of ICls}

Combination of PD-1/PD-L1 and CTLA-4blocking antibodies has the potential to offer synergistic benefits to patients [42]. CCTG CO.26 is a phase II trial of durvalumab (PD-L1 inhibitor) plus tremelimumab (CTLA-4 inhibitor) versus best supportive care (BSC) alone for patients with advanced refractory colorectal cancer (rCRC) (NCT02870920), aiming to evaluate the efficacy and safety of the ICIs combination [43]. With a median follow-up of 15.2 months, the median OS was prolonged by 2.5 months $(6.6$ months for $\mathrm{D}+\mathrm{T}+\mathrm{BSC}$ vs 4.1 months for BSC). CCTG CO.26 is the first study suggesting that anti-PD-L1 plus anti-CTLA-4 may prolong the OS in patients with MSS rCRC.

Preclinical data suggested that the anti-tumor activity of ICIs may be enhanced by inhibiting PGE2 synthesis [44]. In the exploratory study NICHE (NCT03026140), 4/15 (27\%) pathological responses were observed in patients with pMMR tumors, who received ipilimumab plus nivolumab before surgery with or without celecoxib [45]. CD8+PD-1+ T-cell infiltration was predictive of response in pMMR tumors.

Panitumumab, a monoclonal antibody (mAb) targeting the epidermal growth factor receptor (EGFR), is a standard therapy in KRAS/NRAS/BRAF wild-type $\mathrm{mCRC}$, the resistance of which is associated with increased CTLA-4 and PD-L1 expression [46]. LCCC1632 (NCT03442569) is a single-arm phase II clinical trial to investigate the safety and efficacy of nivolumab and ipilimumab with panitumumab in 
patients with KRAS/NRAS/BRAF wild-type MSS refractory mCRC [47]. Among 49 evaluable subjects, 12-week response rate was $35 \%$, with median PFS of 5.7 months [46]. The results demonstrate the safety and efficacy of ICIs combined with anti-EGFR therapy in MSS $\mathrm{mCRC}$, providing merits to further study. All above studies suggested that PD-1 plus CTLA-4 blockade could be a promising treatment strategy for patients with pMMR-MSS CRC, and further larger studies are warranted.

\section{ICls with Radiotherapy}

Preclinical work highlighted that radiotherapy (RT) could cause immunogenic cell death (ICD), which subsequently cause release of damagedassociated molecular patterns (DAMPs), increase of antigen presentation by APCs, priming of T cells and antitumor effects via the abscopal effect [48]. As a hallmark of ICD, DAMPs include cancer-associated neoantigens, inflammatory cytokines and upregulation of immunogenic cell surface markers on tumor cells and stoma.

Although interim results of a single arm phase II study (NCT02437071) reported only 1 of 22 patients of pMMR-MSI-L CRC responded to the combination therapy of pembrolizumab and external beam radiation [49], more encouraging results have been reported successively. In a phase II clinical trial (NCT03104439), dual blockade of CTLA-4 and PD-1 combined with RT yielded a DCR of $29.2 \%(7 / 24)$ and an ORR $12.5 \%(3 / 24)$, respectively [50]. The short-term results of the VOLTAGE-A, a phase I b/II study (NCT02948348), suggested that neoadjuvant chemoradiotherapy (CRT) followed by nivolumab and radical surgery could effectively treat MSS patients with locally advanced rectal cancer (LARC) [41]. Among 37 patients, one patient (3\%) received clinical $C R$ and refused radical surgery after aforementioned treatment, $11(30 \%)$ patients had $\mathrm{pCR}$ (AJCC grade 0 ). The MPR (AJCC grade $0+1$ ) rate was $38 \%(14 / 37)$. These cogent data indicated the huge potential of the treatment strategies of ICIs coupled with RT.

\section{ICls with MEK Inhibitor}

Preclinical data suggested that the inhibition of MEK, a downstream effector of the RAS-MAPK pathway, increased tumor expression of MHC-I and PD-L1, stimulated clonal expansion of peritumoral $\mathrm{T}$ cells, and enhanced anti-tumor activity of ICIs [51, 52].

Accordingly, the combination strategy using the MEK inhibitor cobimetinib and the PD-L1 inhibitor atezolizumab was tested in a phase I b study (NCT01988896) [53, 54]. Preliminary data reported in 2016 showed that 4 of 23 patients with CRC had a partial response (17\%, 3 pMMR-MSI-L, 1 unknown). Follow-up results were presented in 2018, demonstrating manageable adverse events and partial responses in 7 of 84 patients with $\mathrm{mCRC}(8 \% ; 6 \mathrm{MSS} /$ MSI-L, 1 was MSI-H). Although potential synergistic effect was shown, such effect failed to be confirmed in a subsequent phase III study IMblaze370, which is a randomized trial (NCT02788279) using atezolizumab (with or without cobimetinib) versus regorafenib in patients with pMMR-MSI-L rCRC [55]. To adress such unexpected failure, researchers pondered to modify the details and approaches of the combination strategy [56]. Combined MEK inhibitor with ICIs is being tested in several trials $[28,57,58]$.

\section{ICls with anti-angiogenic agents}

Preclinical data suggested that anti-angiogenic agents could increase CD8+ $\mathrm{T}$ cell infiltration and enhance the anti-tumor activity of CD8+ $\mathrm{T}$ cell by upregulating the expression of PD-L1, reducing immunosuppressive cells (TAMs, Tregs), and enhancing interactions between APCs and dendritic cells [59-61].

Based on this theory, a phase I b study (NCT01633970) showed promising results. Of 14 patients with pMMR/MSI-L rCRC received ICI plus anti-angiogenic agents (atezolizumab + bevacizumab), 1 patient had an objective response and 9 patients had stable disease [62, 63]. Recently, encouraging anti-tumor activity from the combination therapy of regorafenib and nivolumab was further confirmed [64]. In REGONIVO (NCT03406871), a phase I b trial, 25 patients with CRC ( 24 pMMR-MSS, 1 dMMR-MSI-H) were enrolled to examine the safety and efficacy of combination of nivolumab and regorafenib. Exciting results demonstrated an ORR of $36 \%$. Median PFS was 7.9 months in CRC, with oneyear PFS and OS rate $41.8 \%$ and $68.0 \%$, respectively. Based on the encouraging data, investigations of larger cohorts are needed.

In LEAP-005 (NCT03797326), a nonrandomized, open-label, phase II study, the efficacy and safety of treatment with combined pembrolizumab and Lenvatinib in patients with previously treated advanced non-MSI-H/pMMR colorectal cancer were preliminarily evaluated [65]. Among 32 patients with a median follow-up of 10.6 months, the ORR and DCR were $22 \%$ and $47 \%$, respectively, with 2.3 -month median PFS and 7.5-month median OS. The median DOR has not been reached. Based on the promising antitumor activity and a manageable safety profile, enrollment was expanded to 100 patients. 


\section{ICls with Bispecific Antibodies}

Bispecific antibodies are a new class of targeted therapeutics designed to bind two different sites on one antigen or two antigens. By targeting two different antigens, it simultaneously bridges the tumor cell and the $\mathrm{T}$ cell, hence enhance the intertumoral $\mathrm{T}$ cell infiltration and activation. CEA-CD3 is the first reported bispecific antibody showing significant efficacy in MSS CRC [66]. CEA-CD3 plus atezolizumab (NCT02650713) revealed more effective clinical activity in patients with metastatic MSS CRC than CEA-CD3 monotherapy (NCT02324257) [67, 68]. In the combination therapy group, the PR was $18 \%(2 / 11)$, and DCR was $82 \%$ $(9 / 11)$ [69].

Another 3 emerging bispecific antibodies, TRAILR2-CDH17 (BI 905711) GCC-CD3 (PF-07062119) and CD137-PD-L1 (FS222), all showed potent T-cell mediated anti-tumor activity in CRC in preclinical trials [70-72]. TRAILR2-CDH17 (BI 905711) was advanced into a Phase $\mathrm{I} a / \mathrm{b}$ clinical trial for patients with advanced gastrointestinal cancers (NCT04137289) in 2019 [73]. As a novel strategy, bispecific antibody needs further investigation.

\section{Other Prospective Combination}

There are some alternative approaches of immune modulation worthy of high anticipation. One promising strategy is combining the immunotherapy with cancer vaccines, which may augment the host antitumor immune response [74]. In a phase II study (NCT02981524) using GVAX colon vaccine (with cyclophosphamide) plus pembrolizumab, biochemical responses $(\geq 30 \%$ decline in CEA) were observed in $7 / 17$ (41\%) patients of pMMR CRC, although it did not meet the primary objective [75]. Continual expansion cohorts are ongoing, and more solid results are anticipated.

Oncolytic viruses (OVs) can cause direct lysis of tumor cells and promote anti-tumor immune response by inducing immunogenic cell death [76]. As the OVs infection can turn the tumor from "cold" to "hot", it enhances the anti-tumor capacity of the ICIs. The efficacy of the combination of OVs with ICIs has been preliminarily confirmed in clinical trials of a wide range of solid tumors [77-80]. In a phase I clinical trial (NCT02636036), enadenotucirev (a chimeric adenovirus) plus nivolumab in solid tumors including CRC is currently being tested [81].

Intestinal bacteria play crucial roles in various fundamental physiopathologic processes [82-84]. Intriguingly, it was recently found that intestinal bacteria Bifidobacterium pseudolongum could significantly affect the efficacy of ICIs, via releasing inosine and subsequently enhancing $\mathrm{T}$ cells activity [85]. Remarkably, in anti-PD-1-refractory metastatic melanoma, the safety and feasibility of the combination of fecal microbiota transplantation (FMT) with ICIs were preliminarily confirmed in a phase I trial (NCT03353402) [86]. These interesting discoveries could probably provide a new ideal strategy for patients with pMMR-MSS CRC.

\section{Cancer vaccines}

Although existing for more than a century, cancer vaccines have barely received response in patients with CRC. Recently, cancer vaccines have elicited renewed interest owing to the convinced efficacy of immunotherapy. Multiple trials aiming to find the right antigenic stimulants are under investigation.

Table 3. Exploring cancer vaccines in colorectal cancer

\begin{tabular}{|c|c|c|c|c|c|c|}
\hline Vector & Clinicaltrials.gov identifier & Phase & Patient population & Treatment/Target & Primary endpoints & Recruitment status \\
\hline \multirow[t]{6}{*}{$\mathrm{DC}$} & NCT01885702 & II & MSI-H CRC & DC vaccine & Safety, feasibility & Active, not recruiting \\
\hline & NCT00103142 & II & $\mathrm{mCRC}$ & PANVAC-V+PANVAC-F+DC & Reference-free survival & Completed \\
\hline & NCT00558051 & I & $\mathrm{mCRC}$ & DC vaccine & Safety, feasibility & Completed \\
\hline & NCT01671592 & I & CRC & Apoptotic autologous tumor-aDC1 & Adverse events & Completed \\
\hline & NCT04147078 & I & CRC & Neoantigen-primed DC & DFS & Recruiting \\
\hline & NCT03730948 & I & CRC & DC vaccine & Safety, immune response & Recruiting \\
\hline \multirow[t]{5}{*}{ Peptide } & NCT03391232 & $\mathrm{I} / \mathrm{II}$ & $\mathrm{mCRC}$ & PolyPEPI1018 & Adverse events & Completed \\
\hline & NCT00228189 & $\mathrm{I} / \mathrm{II}$ & $\mathrm{mCRC}$ & CEA & Immune response & Completed \\
\hline & NCT01461148 & $\mathrm{I} / \mathrm{II}$ & MSI-H CRC & FSP & Immune response & Completed \\
\hline & NCT03689192 & I & $\mathrm{mCRC}$ & ARG1-18, 19, 10 & Adverse events & Recruiting \\
\hline & NCT00641615 & I & CRC & RNF 43-721 & Safety & Completed \\
\hline \multirow[t]{3}{*}{ Virus } & NCT01147965 & $\mathrm{I} / \mathrm{II}$ & Colon cancer & AD5[E1-, E2b-]-CEA(6D) & Safety & Completed \\
\hline & NCT01972737 & I & Colon cancer & Ad5-hGCC-PADRE & $\begin{array}{l}\text { Adverse events, antibody } \\
\text { response }\end{array}$ & Completed \\
\hline & NCT00027354 & I & mCRC & TRICOM-CEA(6D) & Safety, immune response & Completed \\
\hline
\end{tabular}

DC, dendritic cell; MSI-H, microsatellite instability-high; CRC, colorectal cancer; mCRC, metastasis colorectal cancer; DFS, disease-free survival; Ad5-hGCC-PADRE Guanylyl Cyclase C (GCC)-encoding replication-d human type 5 recombinant adenovirus vaccine. 
PolyPEPI1018 consists of 6 synthetic peptides with 12 unique epitopes derived from 7 conserved cancer testis antigens (CTAs) and optimized to induce long lasting CRC specific $\mathrm{T}$ cell responses [87]. In OBERTO (NCT03391232), PolyPEPI1018 was tested in 11 previous untreated patients with MSS MCRC as an add-on to maintenance therapy. 4 patients achieved objective response and/or durable clinical benefit. Broad anticancer immunity was successfully boosted by PolyPEPI1018 at both peripheral and tumor level [88]. Given PolyPEPI1018 promotes infiltration of cytotoxic CD8+ TILs into the core tumor, further study is warranted to investigate the efficacy of combination of PolyPEPI1018 with ICIs in patients with MSS mCRC.

Guanylyl cyclase C (GCC), a membranespanning receptor synthesizing the second messenger cyclic GMP (cGMP), is normally restrictedly expressed by intestinal epithelial cells and a subset of neurons, but universally expressed by metastatic colorectal tumors [89-91]. Ad5-hGCC-PADRE vaccine uses a replication-deficient human type 5 recombinant adenovirus (Ad5) as the vector with GCC fused to the PADRE to induce GCC-specific immune responses. The safety and efficacy were evaluated in a phase I trial (NCT01972737) that enrolled 10 patients with surgically-resected stage 1 or 2 colon cancer [92]. The preliminary results showed that 1 patient $(10 \%)$ had antibody response to GCC and 4 patients $(40 \%)$ exhibited GCC-specific T-cell responses, without significant toxicities [93]. Notably, GCC-specific T-cell response was exclusively cytotoxic $\mathrm{CD}^{+}+$, but not CD4+ helper T cells, which were eliminated by selftolerance [93].

Autologous vaccine OncoVAX is an active specific immunotherapy, which utilizes the patient's own tumor cells containing all relevant tumorassociated antigens to activate the body's immune system to prevent tumor progression after surgery. A randomized phase IIIb trial is being conducted to evaluate the recurrence status of colon cancer following surgery in 500 patients with stage 2 colon cancer after treatment with OncoVAX (NCT02448173) [94].

\section{Adoptive cell therapy}

Another highly anticipated novel treatment to stimulate tumor immunity is adoptive cell therapy (ACT). ACT selects either host cells exhibiting antitumor activity or host cells engineered with chimeric antigens receptors (CARs) or antitumor $\mathrm{T}$ cell receptors (TCR) to augment the host antitumor immune response [95]. Both CAR T therapy and TIL (tumor-infiltrating lymphocyte) therapy have evoked encouraging preliminary results, but the applicability remains to be proved.

The levels of CEA are low or absent in normal cells, but abundant in CRC [96]. Based on this, several trials targeted CEA for ACT. In a phase I trial, CAR T-cells therapy targeting CEA was firstly tested in 3 patients with mCRC [97]. Obvious decreases in serum CEA was observed in all of patients, and one patient received objective response of lung and liver metastasis. Unfortunately, all 3 patients experienced severe colitis. Another phase I trial (NCT02349724) was conducted to evaluate the safety and efficacy of anti-CEA CAR-modified $\mathrm{T}$ cells in CEA positive refractory mCRC patients [98]. 7 of 10 patients obtained stable disease, without significant CARrelated toxicity.

The shed natural killer group 2D (NKG2D) ligands from tumor cells may downregulate NKG2D expression on NK and T cells, contributing to tumor immune escape [99-101]. A novel attempt to further augment the host antitumor immune response is to genetically modify CAR T cells to express proteins such as PD-L1 and NKG2D receptor. The safety and efficacy of this "armored" CRATs remain to be investigated.

Encouraging results on TILs were shown in a case report [102]. Researchers identified a polyclonal CD8+ T-cell response against mutant KRAS G12D in TILs and transferred the TILs into the patient. The result showed that all 7 metastatic lung lesions regressed at the first follow-up of 40 days, and the patient had a 9-month partial response until one lesion had progression. The patient remained 4 months clinically disease-free after the lung resection.

\section{Biomarkers of Response to Immuno- therapy}

Immunotherapy has significantly changed clinical management of CRC. Nevertheless, there is urgent need to explore specific biomarkers to predict responsiveness of immunotherapy. Emerged biomarkers in CRC immunotherapy are classified into 4 main types: tumor mutations, pre-existing immune responses, PD-L1 expression and the microbiota. A limited number of candidate biomarkers in CRC are listed below.

\section{Tumor Mutations}

\section{Tumor mutational burden}

Tumor mutational burden (TMB) refers to the total number of somatic mutations per coding area of a tumor genome, which can measure all nonsynonymous coding mutations in a tumor exome [103, 104]. TMB has been proved to be an independent predictor of therapeutic efficacy of ICIs in several 
solid tumors including CRC [105-107]. As known by now, higher TMB is associated with stronger immunogenicity, which could probably enhance the anti-tumor activity of immunotherapies. Notably, a high TMB value could emerge not only with MSI-H, but also in MSS tumors. The efficacy of immunotherapy was preliminarily confirmed in MSS CRC patients with a high TMB value. In the exploratory analysis of REGONIVO trial, TMB was evaluated in 23 patients with CRC. ORRs were $50 \%$ and $35.3 \%$ in the TMB high and low group, respectively, and the median PFSs were 12.5 vs 7.9 months. Another CCTG CO.26 trial assessed the plasma TMB by analyzing the cfDNA in blood samples. Higher plasma TMB with a cutoff value of 28 per megabase is associated with better OS in MSS CRC patients subjected to the combined PD-L1 and CTLA-4 inhibition. It is suggested that plasma TMB $\geq 28$ may be a biomarker of identifying patients most likely to benefit from durvalumab plus tremelimumab. Undoubtedly, TMB is a great promising biomarker and remains to be further investigated.

\section{POLE/POLD 1}

POLE and POLD1 are crucial for polymerase $\varepsilon$ and $\delta$ encoding, respectively, which are essential for proofreading and fidelity in DNA replication [108, 109]. The somatic or germline mutations in POLE and POLD1 lead to the pathogenesis of CRC via a DNA hypermutated phenotype [110,111]. Nearly $7.4 \%$ of CRCs harbor mutations in either POLE or POLD1 and
$74 \%$ of tumors with POLE or POLD1 mutations were MSS or MSI-L [112]. Among pMMR CRCs, POLEmutant CRCs show prominently higher CD8+ lymphocyte infiltration, expression of cytotoxic T-cell markers and effector cytokines than POLE wild-type CRCs, with upregulated expression levels of PD-L1, PD-1 and CTLA-4, etc. [113]. Considering the enhanced immunogenicity, POLE may become another acceptable effective biomarker similar to MMR/MSI in the near future. NCT03435107, NCT03827044, and NCT03150706 are underway to investigate the benefit of ICIs in POLE-mutant CRC [114-116].

\section{Pre-existing Immune Responses}

Tumor infiltrating lymphocytes (TILs) are associated with improved survival in retrospective studies of CRC patients, particularly for cytotoxic CD8+ T cells [117]. Density and location of the intertumoral $\mathrm{T}$ cells could have a better prognostic value for CRC patients compared with the classical TNM system [118]. Immunoscore is a scoring system evaluating the density of CD3+ T cells and CD8+ T cells both in tumor center and the invasive margin based on standardized criteria. A phase II multicenter study (NCT04262687) is ongoing, aiming to assess the anti-tumor activity of ICIs in combination with chemotherapy and antiangiogenic agents as first-line treatment of pMMR-MSI-L mCRC with high Immunoscore [119].

\section{Potential biomarkers of CRC immunotherapy}

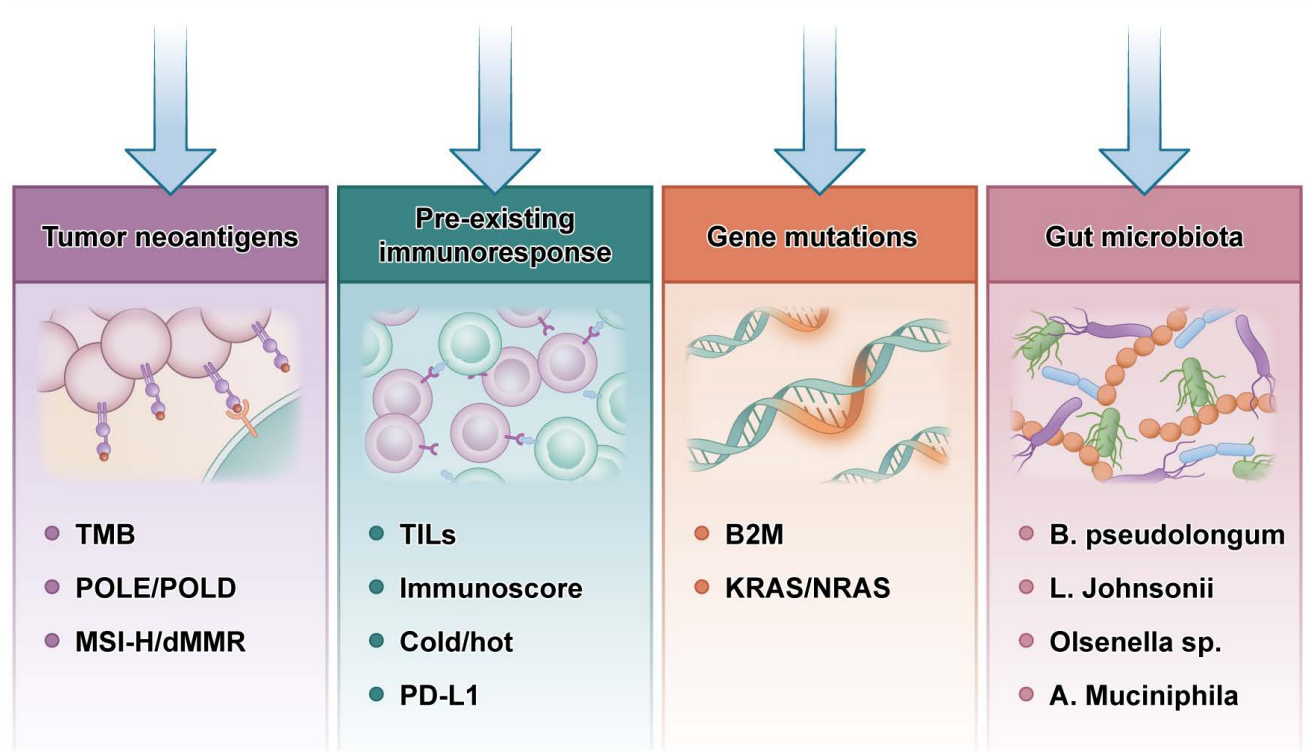

Figure 3. Potential biomarkers of CRC immunotherapy. TMB: tumor mutational burden; MSI-H: microsatellite instability-high; dMMR: mismatch-repair-deficient; TILs: tumor infiltrating lymphocytes; B2M: beta-2-microglobulin; B. pseudolongum: Bifidobacterium pseudolongum; L. johnsonii: Lactobacillus johnsonii; Olsenella sp.:Olsenella species; A. muciniphila: Akkermansia muciniphila. 
Based on the concept of Immunoscore, the classification of tumors was redefined as hot, altered and cold, which are routinely used referring to T cellinfiltrated, inflamed but non-infiltrated, and noninflamed tumors, respectively [120]. The redefinition not only includes Immunoscore, but the tumor immune contexture and microenvironment. The hot tumor could be more sensitive to ICIs, therefore, patients with hot tumors could probably gain more benefits from the immunotherapy. Furthermore, cold tumor can be converted into hot by radiotherapy, chemotherapy and so on. It is incredibly enlightening to develop and validate strategies to overcome primary ICI resistance.

\section{PD-L1 expression}

PD-L1, a co-inhibitory receptor ligand, is recognized as one of the most widely studied biomarkers assayed by immunohistochemical staining, while PD-L1 expression status has not proved to be associated with the efficacy of ICIs in CRC to date. In KEYNOTE016 (NCT01876511), a phase II trial investigating pembrolizumab in patients with refractory $\mathrm{mCRC}$, PFS or OS appeared irrespective of PD-L1 expression level [121]. In Checkmate142 (NCT02060188), another phase II trial investigating the efficacy of monotherapy of nivolumab and combination of nivolumab with ipilimumab, no significant association was found between PD-L1 expression and ORR [122].

\section{The Gut Microbiota}

Gut microbiota have large effect on the efficacy of immunotherapies in multiple cancers, and the composition of the intestinal microbiome could be a potential predictor of the efficacy of ICIs [82-85]. Bifidobacterium pseudolongum, A. muciniphila, Lactobacillus johnsonii and Olsenella species proved to be useful for enhancing the anti-tumor effect of ICIs therapy. Bifidobacterium pseudolongum and A. muciniphila were found to enhance the anti-tumor effect of ICIs therapy through Inosine- $\mathrm{A}_{2 \mathrm{~A}} \mathrm{R}$ signaling [85]. $T$ cell-specific $A_{2 A} R$ signaling could be a promising pathway, through which the gut microbiota shows synergistic effect with immunotherapies. Further studies investigating the mechanisms of the gut microbiota regulating host antitumor immune response are needed.

\section{Conclusions}

Undoubtedly, great progress has been advanced in CRC immunotherapy over the past few years. Recently, FDA granted approval to pembrolizumab and nivolumab (with or without Ipilimumab) for the second-line treatment of patients with dMMR-MSI-H
mCRC based on the cogent data from 2 phase II trials. Based on the compelling data of KEYNOTE177, FDA approved pembrolizumab for the first-line treatment of this subgroup in 2020. Multiple trials exploring potential benefit of ICIs as the first-line treatment for patients with dMMR-MSI-H CRC and those with early-stage dMMR-MSI-H CRC are underway and highly anticipated.

However, the real critical challenge is to find ways to overcome immunotherapy primary resistance in the vast majority of patients with pMMR-MSI-L $\mathrm{mCRC}$. To modulate immune cells and enhance the therapeutic efficacy, various ICIs-based strategies were tested in this subgroup, such as combinational therapy with antibodies blocking PD-1 and CTLA-4, ICIs combined with radiotherapy, ICIs combined with small molecule TKIs such as MEK inhibition and anti-angiogenic agents, and ICIs combined with bispecific antibodies. Although several early-phase trials presented promising data, further studies are warranted to validate the safety and efficacy. Alternative attempts to stimulate tumor immunity and enhance anti-tumor activity including ACT and cancer vaccines are promising areas of active research.

Moreover, biomarkers-based treatment is the inevitable trend of immunotherapy. Selection criteria are indispensable to identify patients who may benefit from these agents. Although some of aforementioned promising biomarkers emerged, discovery and validation of sensitive and specific biomarkers remain as an extremely active area of investigation.

With insights gained from additional trials aiming to develop effective therapeutic strategies, novel combination and biomarkers will likely help to guide clinicians towards a more personalized treatment for CRC patients. It is reasonable to believe that immunotherapy may soon change the treatment landscape for CRC.

\section{Acknowledgements}

\section{Funding}

This study was supported by the National Science Fund for Excellent Young Scholars (No. 81822031) and National Natural Science Foundation of China (No's. 81871913, 81972224, 82073197).

\section{Author Contributions}

A.F. and B.W. performed the literature review, drafted the manuscript; X.W., Y.N., and D.F. contributed to the critical review of the manuscript; Y.L. and X.Z. supervised the study and contributed to the critical editing of the manuscript. All authors have read and agreed to the published version of the manuscript. 


\section{Competing Interests}

The authors have declared that no competing interest exists.

\section{References}

1. Sung H, Ferlay J, Siegel RL, Laversanne M, Soerjomataram I, Jemal A, et al. Global cancer statistics 2020: GLOBOCAN estimates of incidence and mortality worldwide for 36 cancers in 185 countries. CA Cancer J Clin. 2021.

2. Pinsky PF, Doroudi M. Colorectal Cancer Screening. Jama. 2016; 316: 1715.

3. Edwards BK, Ward E, Kohler BA, Eheman C, Zauber AG, Anderson RN, et al. Annual report to the nation on the status of cancer, 1975-2006, featuring colorectal cancer trends and impact of interventions (risk factors, screening, and treatment) to reduce future rates. Cancer. 2010; 116: 544-73.

4. Sargent D, Sobrero A, Grothey A, O'Connell MJ, Buyse M, Andre T, et al. Evidence for cure by adjuvant therapy in colon cancer: observations based on individual patient data from 20,898 patients on 18 randomized trials. J Clin Oncol. 2009; 27: 872-7.

5. Simmonds PC, Primrose JN, Colquitt JL, Garden OJ, Poston GJ, Rees M. Surgical resection of hepatic metastases from colorectal cancer: a systematic review of published studies. Br J Cancer. 2006; 94: 982-99.

6. Tomlinson JS, Jarnagin WR, DeMatteo RP, Fong Y, Kornprat P, Gonen M, et al. Actual 10-year survival after resection of colorectal liver metastases defines cure. J Clin Oncol. 2007; 25: 4575-80.

7. Loupakis F, Cremolini C, Masi G, Lonardi S, Zagonel V, Salvatore L, et al. Initial therapy with FOLFOXIRI and bevacizumab for metastatic colorectal cancer. N Engl J Med. 2014; 371: 1609-18.

8. Hornbech K, Ravn J, Steinbrüchel DA. Outcome after pulmonary metastasectomy: analysis of 5 years consecutive surgical resections 2002-2006. J Thorac Oncol. 2011; 6: 1733-40.

9. Yang Y. Cancer immunotherapy: harnessing the immune system to battle cancer. J Clin Invest. 2015; 125: 3335-7

10. Li GM. Mechanisms and functions of DNA mismatch repair. Cell Res. 2008; 18: 85-98.

11. Ganesh K, Stadler ZK, Cercek A, Mendelsohn RB, Shia J, Segal NH, et al. Immunotherapy in colorectal cancer: rationale, challenges and potential. Nat Rev Gastroenterol Hepatol. 2019; 16: 361-75.

12. Alexander J, Watanabe T, Wu TT, Rashid A, Li S, Hamilton SR. Histopathological identification of colon cancer with microsatellite instability. Am J Pathol. 2001; 158: 527-35.

13. Dolcetti R, Viel A, Doglioni C, Russo A, Guidoboni M, Capozzi E, et al. High prevalence of activated intraepithelial cytotoxic T lymphocytes and increased neoplastic cell apoptosis in colorectal carcinomas with microsatellite instability. Am J Pathol. 1999; 154: 1805-13.

14. Smyrk TC, Watson P, Kaul K, Lynch HT. Tumor-infiltrating lymphocytes are a marker for microsatellite instability in colorectal carcinoma. Cancer. 2001; 91: $2417-22$

15. Gajewski TF, Schreiber $\mathrm{H}, \mathrm{Fu} \mathrm{YX}$. Innate and adaptive immune cells in the tumor microenvironment. Nat Immunol. 2013; 14: 1014-22.

16. Llosa NJ, Cruise M, Tam A, Wicks EC, Hechenbleikner EM, Taube JM, et al. The vigorous immune microenvironment of microsatellite instable colon cancer is balanced by multiple counter-inhibitory checkpoints. Cancer Discov. 2015; 5: 43-51.

17. Boland CR, Thibodeau SN, Hamilton SR, Sidransky D, Eshleman JR, Burt RW, et al. A National Cancer Institute Workshop on Microsatellite Instability for cancer detection and familial predisposition: development of international criteria for the determination of microsatellite instability in colorectal cancer. Cancer Res. 1998; 58: 5248-57.

18. Zaanan A, Shi Q, Taieb J, Alberts SR, Meyers JP, Smyrk TC, et al. Role of Deficient DNA Mismatch Repair Status in Patients With Stage III Colon Cancer Treated With FOLFOX Adjuvant Chemotherapy: A Pooled Analysis From 2 Randomized Clinical Trials. JAMA Oncol. 2018; 4: 379-83.

19. Lenz H-J, Ou F-S, Venook AP, Hochster HS, Niedzwiecki D, Goldberg RM, et al. Impact of consensus molecular subtyping (CMS) on overall survival (OS) and progression free survival (PFS) in patients (pts) with metastatic colorectal cancer (mCRC): Analysis of CALGB/SWOG 80405 (Alliance). 2017; 35: 3511-.

20. Heinemann V, Kraemer N, Buchner H, Weikersthal LFv, Decker T, Kiani A, et al. Somatic DNA mutations, tumor mutational burden (TMB), and MSI Status: Association with efficacy in patients (pts) with metastatic colorectal cancer (mCRC) of FIRE-3 (AIO KRK-0306). 2018; 36: 3591-

21. Tran B, Kopetz S, Tie J, Gibbs P, Jiang ZQ, Lieu CH, et al. Impact of BRAF mutation and microsatellite instability on the pattern of metastatic spread and prognosis in metastatic colorectal cancer. Cancer. 2011; 117: 4623-32.

22. Venderbosch S, Nagtegaal ID, Maughan TS, Smith CG, Cheadle JP, Fisher D, et al. Mismatch repair status and BRAF mutation status in metastatic colorectal cancer patients: a pooled analysis of the CAIRO, CAIRO2, COIN, and FOCUS studies. Clin Cancer Res. 2014; 20: 5322-30.

23. Le DT, Uram JN, Wang H, Bartlett B, Kemberling $H$, Eyring A, et al. Programmed death-1 blockade in mismatch repair deficient colorectal cancer. 2016; 34: 103-

24. Overman MJ, McDermott R, Leach JL, Lonardi S, Lenz HJ, Morse MA, et al. Nivolumab in patients with metastatic DNA mismatch repair-deficient or microsatellite instability-high colorectal cancer (CheckMate 142): an open-label, multicentre, phase 2 study. Lancet Oncol. 2017; 18: 1182-91.

25. Overman MJ, Lonardi S, Wong KYM, Lenz HJ, Gelsomino F, Aglietta M, et al. Durable Clinical Benefit With Nivolumab Plus Ipilimumab in DNA Mismatch Repair-Deficient/Microsatellite Instability-High Metastatic Colorectal Cancer. J Clin Oncol. 2018; 36: 773-9.

26. Andre $\mathrm{T}$, Lonardi $\mathrm{S}$, Wong $\mathrm{M}$, Lenz $\mathrm{H}-\mathrm{J}$, Gelsomino $\mathrm{F}$, Aglietta $\mathrm{M}$, et al Nivolumab + ipilimumab combination in patients with DNA mismatch repair-deficient/microsatellite instability-high (dMMR/MSI-H) metastatic colorectal cancer (mCRC): First report of the full cohort from CheckMate-142. 2018; 36: 553-.

27. NIH-ClinicalTrials.gov. Study of Pembrolizumab (MK-3475) vs Standard Therapy in participants with Microsatellite Instability-High (MSI-H) or Mismatch Repair Deficient (dMMR) Stage IV Colorectal Carcinoma (MK-3475-177/KEYNOTE-177).2021. https://clinicaltrials.gov/ct2/show/ NCT02563002

28. NIH-ClinicalTrials.gov. An Investigational Immuno-therapy Study of Nivolumab, and Nivolumab in combination with other Anti-cancer Drugs, in Colon Cancer That Has Come Back or Has Spread (CheckMate142).2019. https://clinicaltrials.gov/ct2/show/NCT02060188.

29. NIH-ClinicalTrials gov. Combination Chemotherapy, Bevacizumab, and/or Atezolizumab in treating patients with Deficient DNA Mismatch Repair Metastatic Colorectal Cancer, the COMMIT Study.2021. https:// clinicaltrials.gov/ct2/show/NCT02997228.

30. T A, KK S, TW K, BV J, LH J, C P, et al. Study of Pembrolizumab (MK-3475) vs Standard Therapy in Participants With Microsatellite Instability-High (MSI-H) or Mismatch Repair Deficient (dMMR) Stage IV Colorectal Carcinoma (MK-3475-177/KEYNOTE-177). 2021.

31. Andre T, Shiu K-K, Kim TW, Jensen BV, Jensen LH, Punt CJA, et al. Pembrolizumab versus chemotherapy for microsatellite instability-high/ mismatch repair deficient metastatic colorectal cancer: The phase 3 KEYNOTE-177 Study. Journal of Clinical Oncology. 2020; 38: LBA4-LBA.

32. Annals of Oncology. LBA18_PR - Durable clinical benefit with nivolumab (NIVO) plus low-dose ipilimumab (IPI) as first-line therapy in microsatellite instability-high/mismatch repair deficient (MSI-H/dMMR) metastatic colorectal cancer (mCRC). 2018. https://doi.org/10.1093/annonc/mdy424. 019.

33. NIH-ClinicalTrials gov, Combination Chemotherapy, Bevacizumab, and/or Atezolizumab in treating patients with Deficient DNA Mismatch Repair Metastatic Colorectal Cancer, the COMMIT Study.2021. https://clinicaltrials. gov/ct2/show/NCT02997228.

34. NIH-ClinicalTrials.gov. Combination Chemotherapy With or Without Atezolizumab in treating patients with Stage III Colon Cancer and Deficient DNA Mismatch Repair.2021. https://clinicaltrials.gov/ct2/show/ NCT02912559.

35. Sinicrope FA, Ou F-S, Shi Q, Nixon AB, Mody K, Levasseur A, et al. Randomized trial of FOLFOX alone or combined with atezolizumab as adjuvant therapy for patients with stage III colon cancer and deficient DNA mismatch repair or microsatellite instability (ATOMIC, Alliance A021502). 2017; 35: TPS3630-TPS.

36. NIH-ClinicalTrials.gov. Nivolumab, Ipilimumab and COX2-inhibition in Early Stage Colon Cancer: an Unbiased Approach for Signals of Sensitivity (NICHE).2020. https://clinicaltrials.gov/ct2/show/NCT03026140.

37. Chalabi M, Fanchi L, van den Berg J, Beets G, Lopez-Yurda M, Aalbers A, et al. LBA37_PRNeoadjuvant ipilimumab plus nivolumab in early stage colon cancer. Annals of Oncology. 2018; 29.

38. NIH-ClinicalTrials.gov. Veliparib, Pembrolizumab, and Combination Chemotherapy in treating patient with Locally Advanced Rectal Cancer.2021. https://clinicaltrials.gov/ct2/show/NCT02921256.

39. Rahma OE, Yothers G, Hong TS, Russell MM, You YN, Parker W, et al. NRG-GI002: A phase II clinical trial platform using total neoadjuvant therapy (TNT) in locally advanced rectal cancer (LARC)-Pembrolizumab experimental arm (EA) primary results. 2021; 39: 8-

40. NIH-ClinicalTrials.gov. Study to Nivolumab Following Preoperative Chemoradiotherapy.2017.

https://clinicaltrials.gov/ct2/show/NCT02948348.

41. Yuki S, Bando H, Tsukada Y, Inamori K, Komatsu Y, Homma S, et al. Short-term results of VOLTAGE-A: Nivolumab monotherapy and subsequent radical surgery following preoperative chemoradiotherapy in patients with microsatellite stable and microsatellite instability-high locally advanced rectal cancer. 2020; 38: 4100-

42. Parry RV, Chemnitz JM, Frauwirth KA, Lanfranco $A R$, Braunstein $I_{\text {, }}$ Kobayashi SV, et al. CTLA-4 and PD-1 receptors inhibit T-cell activation by distinct mechanisms. Mol Cell Biol. 2005; 25: 9543-53.

43. Chen EX, Jonker DJ, Loree JM, Kennecke HF, Berry SR, Couture F, et al. Effect of Combined Immune Checkpoint Inhibition vs Best Supportive Care Alone in Patients With Advanced Colorectal Cancer: The Canadian Cancer Trials Group CO.26 Study. JAMA Oncol. 2020; 6: 831-8.

44. Zelenay S, van der Veen AG, Böttcher JP, Snelgrove KJ, Rogers N, Acton SE, et al. Cyclooxygenase-Dependent Tumor Growth through Evasion of Immunity. Cell. 2015; 162: 1257-70.

45. Chalabi M, Fanchi LF, Dijkstra KK, Van den Berg JG, Aalbers AG, Sikorska K, et al. Neoadjuvant immunotherapy leads to pathological responses in MMR-proficient and MMR-deficient early-stage colon cancers. Nat Med. 2020; 26: $566-76$ 
46. Lee MS, Loehrer PJ, Imanirad I, Cohen S, Ciombor KK, Moore DT, et al. Phase II study of ipilimumab, nivolumab, and panitumumab in patients with KRAS/NRAS/BRAF wild-type (WT) microsatellite stable (MSS) metastatic colorectal cancer (mCRC). 2021; 39: 7-

47. NIH-ClinicalTrials.gov. PhII Trial Panitumumab, Nivolumab, Ipilimumab in Kras/Nras/BRAF Wild-type MSS Refractory mCRC.2020. https:// clinicaltrials.gov/ct2/show/NCT03442569.

48. McLaughlin M, Patin EC, Pedersen M, Wilkins A, Dillon MT, Melcher AA, et al. Inflammatory microenvironment remodelling by tumour cells after radiotherapy. Nat Rev Cancer. 2020; 20: 203-17.

49. Segal N, Kemeny N, Cercek A, Reidy D, Raasch P, Warren P, et al. Non-randomized phase II study to assess the efficacy of pembrolizumab (Pem) plus radiotherapy (RT) or ablation in mismatch repair proficient (pMMR) metastatic colorectal cancer (mCRC) patients. Journal of Clinical Oncology. 2016; 34: 3539-

50. Parikh AR, Clark JW, Wo JY-L, Yeap BY, Allen JN, Blaszkowsky LS, et al. A phase II study of ipilimumab and nivolumab with radiation in microsatellite stable (MSS) metastatic colorectal adenocarcinoma (mCRC). 2019; 37: 3514-

51. Rosen LS, LoRusso P, Ma WW, Goldman JW, Weise A, Colevas AD, et al. A first-in-human phase I study to evaluate the MEK1/2 inhibitor, cobimetinib, administered daily in patients with advanced solid tumors. Invest New Drugs. 2016; 34: 604-13.

52. Ebert PJR, Cheung J, Yang Y, McNamara E, Hong R, Moskalenko M, et al. MAP Kinase Inhibition Promotes $\mathrm{T}$ Cell and Anti-tumor Activity in Combination with PD-L1 Checkpoint Blockade. Immunity. 2016; 44: 609-21.

53. Bendell JC, Kim TW, Goh BC, Wallin J, Oh D-Y, Han S-W, et al. Clinical activity and safety of cobimetinib (cobi) and atezolizumab in colorectal cancer (CRC). 2016; 34: 3502-

54. Bendell JC, Bang Y-J, Chee CE, Ryan DP, McRee AJ, Chow LQ, et al. A phase Ib study of safety and clinical activity of atezolizumab (A) and cobimetinib (C) in patients (pts) with metastatic colorectal cancer (mCRC). 2018; 36: 560-

55. Eng C, Kim TW, Bendell J, Argilés G, Tebbutt NC, Di Bartolomeo M, et al. Atezolizumab with or without cobimetinib versus regorafenib in previously treated metastatic colorectal cancer (IMblaze370): a multicentre, open-label, phase 3, randomised, controlled trial. Lancet Oncol. 2019; 20: 849-61.

56. Sclafani F. MEK and PD-L1 inhibition in colorectal cancer: a burning blaze turning into a flash in the pan. Lancet Oncol. 2019; 20: 752-3.

57. NIH-ClinicalTrials.gov. Study of Durvalumab (MEDI4736) (Anti-PD-L1) and Trametinib (MEKi) in MSS Metastatic Colon Cancer.2020. https:// clinicaltrials.gov/ct2/show/NCT03428126.

58. NIH-ClinicalTrials.gov. Study of Binimetinib + Nivolumab Plus or Minus Ipilimumab in patients with Previously Treated Microsatellite-stable (MSS) Metastatic Colorectal Cancer with RAS Mutation.2021. https://clinicaltrials. gov/ct2/show/NCT03271047.

59. Davis PJ, Mousa SA. Chapter 8 - Tyrosine Kinase Inhibitors and Angiogenesis. In: Mousa SA, Davis PJ, editors. Anti-Angiogenesis Strategies in Cancer Therapeutics. Boston: Academic Press; 2017. p. 125-31.

60. Mousa SA, Muralidharan-Chari V, Davis PJ. Chapter 4 - Interface between Thrombosis, Inflammation, and Angiogenesis in Cancer Progression. In: Mousa SA, Davis PJ, editors. Anti-Angiogenesis Strategies in Cancer Therapeutics. Boston: Academic Press; 2017. p. 51-68.

61. Hodi FS, Lawrence D, Lezcano C, Wu X, Zhou J, Sasada T, et al. Bevacizumab plus ipilimumab in patients with metastatic melanoma. Cancer Immunol Res. 2014; 2: 632-42.

62. Hochster HS, Bendell JC, Cleary JM, Foster P, Zhang W, He X, et al. Efficacy and safety of atezolizumab (atezo) and bevacizumab (bev) in a phase Ib study of microsatellite instability (MSI)-high metastatic colorectal cancer (mCRC). 2017; 35: 673-

63. Bendell JC, Powderly JD, Lieu CH, Eckhardt SG, Hurwitz H, Hochster HS, et al. Safety and efficacy of MPDL3280A (anti-PDL1) in combination with bevacizumab (bev) and/or FOLFOX in patients (pts) with metastatic colorectal cancer (mCRC). 2015; 33: 704-

64. Fukuoka S, Hara H, Takahashi N, Kojima T, Kawazoe A, Asayama M, et al. Regorafenib Plus Nivolumab in Patients With Advanced Gastric or Colorectal Cancer: An Open-Label, Dose-Escalation, and Dose-Expansion Phase Ib Trial (REGONIVO, EPOC1603). J Clin Oncol. 2020; 38: 2053-61.

65. Gomez-Roca C, Yanez E, Im S-A, Alvarez EC, Senellart H, Doherty M, et al. LEAP-005: A phase II multicohort study of lenvatinib plus pembrolizumab in patients with previously treated selected solid tumors-Results from the colorectal cancer cohort. Journal of Clinical Oncology. 2021; 39: 94-

66. Tabernero J, Melero I, Ros W, Argiles G, Marabelle A, Rodriguez-Ruiz ME, et al. Phase Ia and $\mathrm{Ib}$ studies of the novel carcinoembryonic antigen (CEA) T-cell bispecific (CEA CD3 TCB) antibody as a single agent and in combination with atezolizumab: Preliminary efficacy and safety in patients with metastatic colorectal cancer (mCRC). 2017; 35: 3002-.

67. NIH-ClinicalTrials.gov. A Study of the Safety, Pharmacokinetics, and Therapeutic Activity of RO6958688 in combination with Atezolizumab in participants with Locally Advanced and/or Metastatic Carcinoembryonic Antigen (CEA)-Positive Solid Tumors.2020. https://clinicaltrials.gov/ct2/ show/NCT02650713.

68. NIH-ClinicalTrials.gov. A Study of RO6958688 in participants with Locally Advanced and/or Metastatic Carcinoembryonic Antigen Positive Solid Tumors.2020. https://clinicaltrials.gov/ct2/show/NCT02324257.
69. al SNe. Phase-I studies of the novel carcinoembryonic antigen $\mathrm{T}$ cell bispecific (CEACD3 TCB) antibody as a single agent and in combination with atezolizumab. Ann Oncol. 2017; 28: 122-41.

70. García-Martínez JM, Wang S, Weishaeupl C, Wernitznig A, Chetta P, Pinto C, et al. Selective Tumor Cell Apoptosis and Tumor Regression in CDH17-Positive Colorectal Cancer Models using BI 905711, a Novel Liver-Sparing TRAILR2 Agonist. 2020.

71. Mathur D, Root AR, Bugaj-Gaweda B, Bisulco S, Tan X, Fang W, et al. A Novel GUCY2C-CD3 T-Cell Engaging Bispecific Construct (PF-07062119) for the Treatment of Gastrointestinal Cancers. Clin Cancer Res. 2020; 26: 2188-202.

72. Lakins MA, Koers A, Giambalvo R, Munoz-Olaya J, Hughes R, Goodman E, et al. FS222, a CD137/PD-L1 Tetravalent Bispecific Antibody, Exhibits Low Toxicity and Antitumor Activity in Colorectal Cancer Models. Clin Cancer Res. 2020; 26: 4154-67.

73. NIH-ClinicalTrials.gov. A First-in-human Phase Ia/b, Open Label, Multicentre, Dose Escalation Study of BI 905711 in patients with Advanced Gastrointestinal Cancers.2021. https://clinicaltrials.gov/ct2/show/ NCT04137289.

74. Johnson BA, Yarchoan M, Lee V, Laheru DA, Jaffee EM. Strategies for Increasing Pancreatic Tumor Immunogenicity. 2017; 23: 1656-69.

75. Yarchoan M, Huang CY, Zhu Q, Ferguson AK, Durham JN, Anders RA, et al. A phase 2 study of GVAX colon vaccine with cyclophosphamide and pembrolizumab in patients with mismatch repair proficient advanced colorectal cancer. Cancer Med. 2020; 9: 1485-94.

76. Ribas A, Dummer R, Puzanov I, VanderWalde A, Andtbacka RHI, Michielin $\mathrm{O}$, et al. Oncolytic Virotherapy Promotes Intratumoral T Cell Infiltration and Improves Anti-PD-1 Immunotherapy. Cell. 2017; 170: 1109-19.e10.

77. Bourgeois-Daigneault MC, Roy DG, Aitken AS, El Sayes N, Martin NT, Varette $\mathrm{O}$, et al. Neoadjuvant oncolytic virotherapy before surgery sensitizes triple-negative breast cancer to immune checkpoint therapy. Sci Transl Med. 2018; 10.

78. Pearl TM, Markert JM, Cassady KA, Ghonime MG. Oncolytic Virus-Based Cytokine Expression to Improve Immune Activity in Brain and Solid Tumors. Mol Ther Oncolytics. 2019; 13: 14-21.

79. Samson A, Scott KJ, Taggart D, West EJ, Wilson E, Nuovo GJ, et al Intravenous delivery of oncolytic reovirus to brain tumor patients immunologically primes for subsequent checkpoint blockade. Sci Transl Med. 2018; 10.

80. Chaurasiya S, Warner S. Viroimmunotherapy for Colorectal Cancer: Clinical Studies. 2017; 5: 11

81. NIH-ClinicalTrials.gov. Phase I Study of Enadenotucirev and PD-1 Inhibitor in Subjects With Metastatic or Advanced Epithelial Tumors (SPICE).2020. https://clinicaltrials.gov/ct2/show/NCT02636036.

82. Sivan A, Corrales L, Hubert N, Williams JB, Aquino-Michaels K, Earley ZM, et al. Commensal Bifidobacterium promotes antitumor immunity and facilitates anti-PD-L1 efficacy. Science. 2015; 350: 1084-9.

83. Vétizou M, Pitt JM, Daillère R, Lepage $P$, Waldschmitt N, Flament $C$, et al. Anticancer immunotherapy by CTLA-4 blockade relies on the gut microbiota. Science. 2015; 350: 1079-84

84. Routy B, Le Chatelier E, Derosa L, Duong CPM, Alou MT, Daillère R, et al. Gut microbiome influences efficacy of PD-1-based immunotherapy against epithelial tumors. Science. 2018; 359: 91-7.

85. Mager LF, Burkhard R, Pett N, Cooke NCA, Brown K, Ramay H, et al. Microbiome-derived inosine modulates response to checkpoint inhibitor immunotherapy. Science (New York, NY). 2020; 369: 1481-9.

86. Baruch EN, Youngster I, Ben-Betzalel G, Ortenberg R, Lahat A, Katz L, et al. Fecal microbiota transplant promotes response in immunotherapy-refractory melanoma patients. Science. 2021; 371: 602-9.

87. NIH-ClinicalTrials.gov. PolyPEPI1018 Vaccine and CDx for the Treatment of Metastatic Colorectal Cancer (OBERTO) (OBERTO).2020. https:// clinicaltrials.gov/ct2/show/NCT03391232.

88. Hubbard JM, Cremolini C, Graham RP, Moretto R, Mitchell JL, Wessling J, et al. Evaluation of safety, immunogenicity, and preliminary efficacy of PolyPEPI1018 off-the-shelf vaccine with fluoropyrimidine/bevacizumab maintenance therapy in metastatic colorectal cancer (mCRC) patients. 2020; 38: 4048

89. Carrithers SL, Barber MT, Biswas S, Parkinson SJ, Park PK, Goldstein SD, et al. Guanylyl cyclase $\mathrm{C}$ is a selective marker for metastatic colorectal tumors in human extraintestinal tissues. Proc Natl Acad Sci U S A. 1996; 93: 14827-32.

90. Lucas KA, Pitari GM, Kazerounian S, Ruiz-Stewart I, Park J, Schulz S, et al. Guanylyl cyclases and signaling by cyclic GMP. Pharmacol Rev. 2000; 52: 375-414.

91. Gong R, Ding C, Hu J, Lu Y, Liu F, Mann E, et al. Role for the membrane receptor guanylyl cyclase- $\mathrm{C}$ in attention deficiency and hyperactive behavior. Science. 2011; 333: 1642-6.

92. NIH-ClinicalTrials.gov. Phase I Study of Ad5-hGCC (Human Guanylyl Cyclase C)-PADRE in Stage I/II Colon Cancer.2017. https://clinicaltrials. gov/ct2/show/NCT01972737.

93. Snook AE, Baybutt TR, Xiang B, Abraham TS, Flickinger JC, Jr., Hyslop T, et al. Split tolerance permits safe Ad5-GUCY2C-PADRE vaccine-induced T-cell responses in colon cancer patients. J Immunother Cancer. 2019; 7: 104.

94. NIH-ClinicalTrials.gov. A Multicenter Study of Active Specific Immunotherapy with OncoVax ${ }^{\circledR}$ in patients with Stage II Colon Cancer.2015. https://clinicaltrials.gov/ct2/show/NCT02448173. 
95. Rosenberg SA, Restifo NP. Adoptive cell transfer as personalized immunotherapy for human cancer. Science. 2015; 348: 62-8.

96. Hammarström S. The carcinoembryonic antigen (CEA) family: structures, suggested functions and expression in normal and malignant tissues. Semin Cancer Biol. 1999; 9: 67-81.

97. Parkhurst MR, Yang JC, Langan RC, Dudley ME, Nathan DA, Feldman SA, et al. $\mathrm{T}$ cells targeting carcinoembryonic antigen can mediate regression of metastatic colorectal cancer but induce severe transient colitis. Mol Ther. 2011; 19: 620-6.

98. Katz SC, Burga RA, McCormack E, Wang LJ, Mooring W, Point GR, et al. Phase I Hepatic Immunotherapy for Metastases Study of Intra-Arterial Chimeric Antigen Receptor-Modified T-cell Therapy for CEA+ Liver Metastases. Clin Cancer Res. 2015; 21: 3149-59.

99. Holdenrieder S, Stieber P, Peterfi A, Nagel D, Steinle A, Salih HR Soluble MICB in malignant diseases: analysis of diagnostic significance and correlation with soluble MICA. Cancer Immunol Immunother. 2006; 55: 1584-9.

100. Salih HR, Rammensee HG, Steinle A. Cutting edge: down-regulation of MICA on human tumors by proteolytic shedding. J Immunol. 2002; 169: 4098-102.

101. Lonez C, Verma B, Hendlisz A, Aftimos P, Awada A, Van Den Neste E, et al. Study protocol for THINK: a multinational open-label phase I study to assess the safety and clinical activity of multiple administrations of NKR-2 in patients with different metastatic tumour types. BMJ Open. 2017; 7: e017075.

102. Tran E, Robbins PF, Lu YC, Prickett TD, Gartner JJ, Jia L, et al. T-Cell Transfer Therapy Targeting Mutant KRAS in Cancer. N Engl J Med. 2016; 375: 2255-62.

103. Yarchoan M, Albacker LA, Hopkins AC, Montesion M, Murugesan K, Vithayathil TT, et al. PD-L1 expression and tumor mutational burden are independent biomarkers in most cancers. JCI Insight. 2019; 4.

104. Fancello L, Gandini S, Pelicci PG, Mazzarella L. Tumor mutational burden quantification from targeted gene panels: major advancements and challenges. J Immunother Cancer. 2019; 7: 183.

105. Yarchoan M, Hopkins A, Jaffee EM. Tumor Mutational Burden and Response Rate to PD-1 Inhibition. N Engl J Med. 2017; 377: 2500-1.

106. Cristescu R, Mogg R, Ayers M, Albright A, Murphy E, Yearley J, et al. Pan-tumor genomic biomarkers for PD-1 checkpoint blockade-based immunotherapy. Science. 2018; 362.

107. Panda A, Betigeri A, Subramanian K, Ross JS, Pavlick DC, Ali S, et al. Identifying a Clinically Applicable Mutational Burden Threshold as a Potential Biomarker of Response to Immune Checkpoint Therapy in Solid Tumors. JCO Precis Oncol. 2017; 2017.

108. Pursell ZF, Isoz I, Lundström E-B, Johansson E, Kunkel TA. Yeast DNA Polymerase $\varepsilon$ Participates in Leading-Strand DNA Replication. Science. 2007; 317: 127-30.

109. Rayner E, van Gool IC, Palles C, Kearsey SE, Bosse T, Tomlinson I, et al. A panoply of errors: polymerase proofreading domain mutations in cancer. Nat Rev Cancer. 2016; 16: 71-81.

110. Palles C, Cazier JB, Howarth KM, Domingo E, Jones AM, Broderick P, et al. Germline mutations affecting the proofreading domains of POLE and POLD1 predispose to colorectal adenomas and carcinomas. Nat Genet. 2013; 45: 136-44.

111. Li HD, Cuevas I, Zhang M, Lu C, Alam MM, Fu YX, et al. Polymerasemediated ultramutagenesis in mice produces diverse cancers with high mutational load. J Clin Invest. 2018; 128: 4179-91.

112. Wang F, Zhao Q, Wang YN, Jin Y, He MM, Liu ZX, et al. Evaluation of POLE and POLD1 Mutations as Biomarkers for Immunotherapy Outcomes Across Multiple Cancer Types. JAMA Oncol. 2019; 5: 1504-6.

113. Domingo E, Freeman-Mills L, Rayner E, Glaire M, Briggs S, Vermeulen L, et al. Somatic POLE proofreading domain mutation, immune response, and prognosis in colorectal cancer: a retrospective, pooled biomarker study. Lancet Gastroenterol Hepatol. 2016; 1: 207-16.

114. NIH-ClinicalTrials.gov. Durvalumab for MSI-H or POLE Mutated Metastatic Colorectal Cancer.2020. https://clinicaltrials.gov/ct2/show/NCT03435107.

115. NIH-ClinicalTrials.gov. Avelumab Plus 5-FU Based Chemotherapy as Adjuvant Treatment for Stage 3 MSI-High or POLE Mutant Colon Cancer (POLEM).2021. https://clinicaltrials.gov/ct2/show/NCT03827044.

116. NIH-ClinicalTrials.gov. Avelumab for MSI-H or POLE Mutated Metastatic Colorectal Cancer.2020. https://clinicaltrials.gov/ct2/show/NCT03150706.

117. Huh JW, Lee JH, Kim HR. Prognostic Significance of Tumor-Infiltrating Lymphocytes for patients with Colorectal Cancer. Archives of Surgery. 2012; 147: 366-72.

118. Pagès F, Mlecnik B, Marliot F, Bindea G, Ou FS, Bifulco C, et al. International validation of the consensus Immunoscore for the classification of colon cancer: a prognostic and accuracy study. Lancet. 2018; 391: 2128-39.

119. NIH-ClinicalTrials.gov. Chemotherapy and Immunotherapy as Treatment for MSS Metastatic Colorectal Cancer with High Immune Infiltrate (POCHI).2020. https://clinicaltrials.gov/ct2/show/NCT04262687.

120. Galon J, Bruni D. Approaches to treat immune hot, altered and cold tumours with combination immunotherapies. Nat Rev Drug Discov. 2019; 18: 197-218.

121. Le DT, Uram JN, Wang H, Bartlett BR, Kemberling H, Eyring AD, et al. PD-1 Blockade in Tumors with Mismatch-Repair Deficiency. N Engl J Med. 2015; 372: 2509-20.

122. Overman MJ LS, Leone F, et al. Nivolumab in patients with DNA mismatch repair deficient/microsatellite instability high metastatic colorectal cancer: update from CheckMate 142. . In: American Society of Clinical Oncology Annual Meeting Chicago: American Society of Clinical Oncology. 2017. 